\title{
Partial anterior mitral leaflet resection with adjacent ring plication - feasibility pilot study
}

\author{
PAWEŁ ANTOSIK**, TORNIKE SOLOGASHWILLI*, MAŁGORZATA PAWELEC-WOJTALIK***, \\ ANDRZEJ WODZIŃSKI, PIOTR ŁADZIŃSKI, ALICJA BARTKOWSKA-ŚNIATKOWSKA****, \\ DOROTA BUKOWSKA**, MICHAŁ SOBIERAJ, TOMASZ NAŁĘCZ, EDYTA CUDAK, \\ MICHAŁ WOJTALIK
}

\begin{abstract}
Department of Pediatric Cardiac Surgery, Poznan University of Medical Sciences, Szpitalna 27/33, 60-578 Poznan, Poland *Department of Cardiac Surgery, Jo Ann Medical Centre, 21, Ljubljana str., Tbilisi 0159, Georgia ${ }^{* *}$ Institute of Veterinary Sciences, the Faculty of Veterinary Medicine and Animal Sciences, Poznan University of Life Sciences, Wolynska 35, 60-637 Poznan, Poland

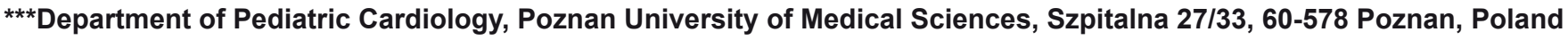

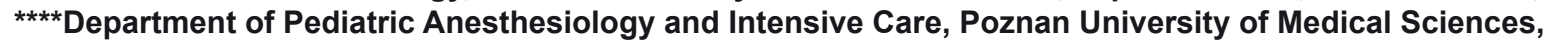
Szpitalna 27/33, 60-578 Poznan, Poland
\end{abstract} Antosik P., Sologashwilli T., Pawelec-Wojtalik M., Wodziński A., Ładziński P., Bartkowska-Śniatkowska A.,
Bukowska D., Sobieraj M., Nałęcz T., Cudak E., Wojtalik M.

Partial anterior mitral leaflet resection with adjacent ring plication - feasibility pilot study

\section{Summary}

The aim of the study was to assess the feasibility and repeatability of a partial removal of the anterior mitral leaflet medial part (AML-A3) accompanied by corresponding anterior mitral annulus plication as a method of valve repair. Four male land race pigs underwent median sternotomy and consecutive cardio-pulmonary by-pass under general anesthesia with hemodynamic monitoring. The AML was detached from the annulus at $1 / 3$ of its length beginning from the postero-median commissure in the direction to the aortic valve and cut off towards the A2-A3 indentation. Three single 3-0 silk sutures were utilized to plicate the mitral annulus segment corresponding with the detached leaflet. The remaining free edge of the AML lateral part was reattached to the plicated ring and to the postero-median commissure with the running 5-0 polypropylene suture. Preoperative and postoperative epicardial echocardiography was performed. All animals survived the operation and were weaned from the cardiopulmonary bypass. All reconstructed MVs were competent. Mean MV diameter decreased after the plasty and did not cause significant diastolic MV flow velocity increase. LVOT anatomy was not altered as well as LVOT flow velocities remained unchanged. The AoV was competent after the operation and did not change its diameter. Partial AML resection completed by corresponding annulus plication is a feasible and repeatable procedure. It does not negatively influence the functioning of LVOT and AoV. Infective and/or congenital MV involvement in selected patients could be a possible indication for this method of MV reconstruction.

Keywords: mitral valve, AML, ring plication, operation

Valve replacement seems to be the most straightforward option for patients with mitral valve (MV) pathology. However, this approach is connected with all the intrinsic side effects of mechanical or biological valve implantation, including susceptibility to infection, thrombosis and degeneration. Thus, a reconstructive surgery has been recognized as the best option for the majority of patients including children. Many repair techniques have been developed to address mitral valve insufficiency (MI) in case of congenital, ischemic and infective pathologies. The majority of procedures concern the posterior mitral leaflet (PML) and the posterior annulus as well as commissures.
The techniques regarding anterior mitral leaflet (AML) and anterior annulus are less frequently utilized in clinical practice as there is a concern of possible distortion of the aortic valve, narrowing of left ventricle outflow tract and unpredictable long-term MV function. Nevertheless, certain interventions on AML were introduced with acceptable results to treat MV systolic anterior motion, degenerative MV disease or Barlow disease $(3,7)$.

Infective and congenital pathologies in children necessitate a search for an alternative reconstructive approach when all procedures utilizing implants (e.g. artificial chordae, pericardial patches) or an opera- 
A

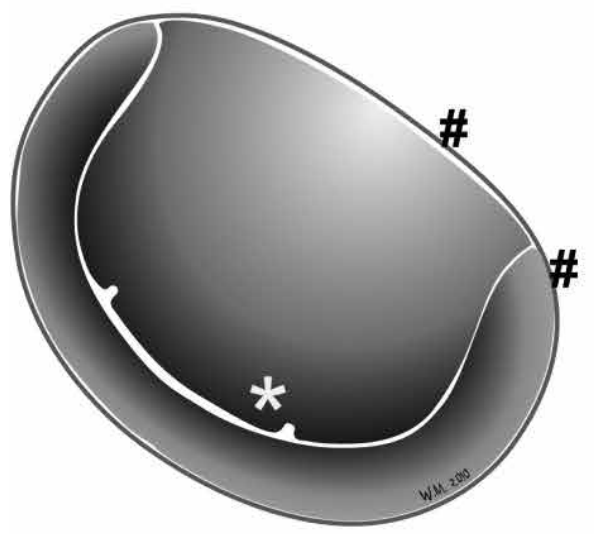

B

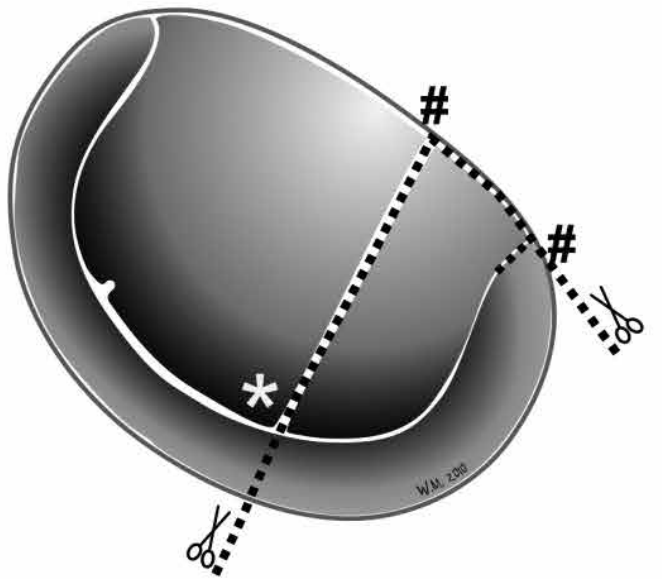

C

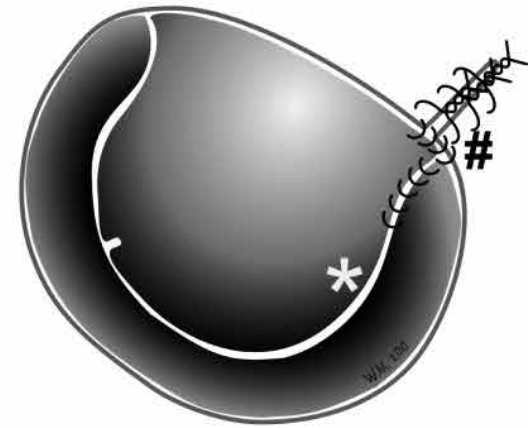

Fig. 1. Schematic illustration of the surgical technique

Explanations: A - mitral valve before the procedure; B - detachment of A3 segment from the annulus and the remaining part of the anterior mitral leaflet and its removal; $\mathrm{C}$ - plication of the anterior part of the annulus and reattachment of the remaining part of the anterior mitral leaflet; \#-\#- plicated segment of mitral valve anterior annulus; * - indentation between A2 and A3 scallop

tion concerning the posterior part of the annulus and adjacent leaflet (applied successfully in adults) are contraindicated. This may be especially true when an MV lesion concerns only a part of the AML in a small child. Thus, the aim of this study was to investigate the feasibility and repeatability of the partial removal of the medial part of AML (A3 scallop) accompanied by corresponding anterior MV annulus plication in order to correct MI.

\section{Material and methods}

Animals and surgical procedure. Four male land race pigs weighting meanly $70 \mathrm{~kg}$ (range $68-73 \mathrm{~kg}$ ) were utilized. They were premedicated with $3 \mathrm{mg} / \mathrm{kg}$ azaperone (Stresnil - Janssen Pharmaceutica) and consecutively anaesthetized with $2 \%$ isoflurane (Isoflurane - Abbott), $4 \mathrm{mg}$ pancuronium bromide (Pavulon - Organon) and $0.5 \mathrm{mcg} /$ $\mathrm{kg} / \mathrm{min}$ fentanyl (Fentanyl - Sintetica) and intubated. The animals were ventilated with a Servo Ventilator 900D respirator (Siemens, Germany) as well as monitored by the use of 3-lead ECG, SpO2, temperature and direct arterial and central venous blood pressures measurement (AS/ 3 cardiomonitor - Datex, Finland). The animals were heparinized (initial dose $15.000 \mathrm{IU}$ ) in order to achieve ACT values between 400 and 450 seconds throughout all the procedure (Hemochrone, Datex). The central arterial and venous lines were introduced by dissection of the right groin vessels. A median sternotomy was performed with the use of heavy scissors. It was followed by longitudinal pericardiotomy and exposition of the heart.

Normothermic extracorporeal circulation was accomplished by cannulation of the ascending aorta and right atrial appendage $(15 \mathrm{~F}$ - aortic, $22 \mathrm{~F}$ - venous, Apex HP oxygenator - Sorin). The aorta was cross-clamped and St. Thomas cold crystalloid cardioplegia was administered to its root during 5 minutes. The left atrium was opened and mitral valve exposed with the use of mitral retractors. AML, PML as well as antero-lateral and postero-median commissures were identified. The anterior leaflet was detached from annulus approximately at $1 / 3$ of its attachment's lengths beginning from the postero-median commissure in direction to the aortic valve and cut off towards A2-A3 indentation - Fig. 1b. Three single 3-0 silk sutures were utilized to plicate mitral annulus segment corresponding with the detached leaflet. The free edge of the AML remaining part was reattached to the plicated ring and to the posteromedian commissure with the running 5-0 polypropylene suture - Fig. 1c. The water test was performed to verify the competence of the valve. The left atrium was closed with a running 5-0 polypropylene suture. After de-airing of the heart the aortic clamp was released. All animals required defibrillations and lidocaine administrations $(1 \mathrm{mg} / \mathrm{kg})$ to treat ventricular fibrillation after the declamping of the aorta. After restoration of the sinus rhythm, animals were weaned from the extracorporeal circulation after the introduction of catecholamine support by dobutamine $(10 \mathrm{mcg} / \mathrm{kg} / \mathrm{min})$.

At the end of the experiment all animals were sacrificed by potassium chloride overdose. The experimental protocol was approved by the Animal Experimentation Ethical Committee of the Poznań Medical University (authorization 2/2009) and carried out in conformance with the Guide for Care and Use of the Laboratory Animals (National Research Council, Washington, DC: National Academy Press; 1996).

Echocardiographic study. Epicardial echocardiography (ALOKA ProFound SSD-Alfa 7 with 1-5 MHz probe) was performed before and after the operation when a stable hemodynamics was achieved. The following parameters were registered: MV lateral diameter, MV regurgitation, MV diastolic flow velocity, flow velocity in the left ventricular outflow tract (LVOT), aortic valve (AoV) regurgitation and diameter. Valve insufficiency was assessed in a 4 grade scale: no regurgitation, trivial, moderate and severe.

Statistical analysis. Preoperative and postoperative data was compared with the use of nonparametric sing test. $p$-value less than 0.05 was considered statistically significant. 


\section{Results and discussion}

No heart structure anomaly was noticed preoperatively by direct inspection and by echocardiography. All animals survived the operation and were weaned from the cardiopulmonary bypass. No intraoperative complication was observed. Plication of the anterior MV annulus was carried out easily without application of important tension while approximating annular tissue. The reconstructed MVs were competent and both leaflets showed good mobility during the cardiac cycle. The results of echocardiograpic measurements are presented in Tab. 1 and 2. Mitral valve diameter after MV plasty decreased without reaching statistical significance and did not cause significant diastolic MV flow velocity increase. LVOT anatomy was not altered

Tab. 1. Echocardiographic MV assessment before and after the procedure

\begin{tabular}{|l|c|c|c|c|c|c|}
\hline \multicolumn{1}{|c|}{ Parameter } & Animal & \multicolumn{2}{|c|}{ Before the plasty } & \multicolumn{2}{|c|}{ After the plasty } & \multirow{2}{*}{ v-value } \\
& & value & median & value & median & \\
\hline $\begin{array}{l}\text { MV lateral diameter } \\
\text { (mm) }\end{array}$ & 1 & 35 & & 29 & & \\
& 2 & 36 & & 28 & & \\
& 4 & 42 & & 27 & & \\
Diastolic MV maximal & 1 & 0.74 & & 1.48 & & \\
flow velocity - wave E \\
(m/s)
\end{tabular}

Explanation: $\mathrm{MV}$ - mitral valve

Tab. 2. Echocardiographic assessment of LVOT and AoV before and after the procedure

\begin{tabular}{|l|c|c|c|c|c|c|}
\hline \multirow{2}{*}{ Parameter } & \multirow{2}{*}{ Animal } & \multicolumn{2}{|c|}{ Pre op. } & \multicolumn{2}{c|}{ Post op. } & \multirow{2}{*}{ p-value } \\
\hline AoV diameter (mm) & 1 & 24 & & 23 & & \\
& 2 & 23 & & 22 & & \\
& 3 & 23 & & 22 & & \\
& 4 & 21 & & 19 & & \\
LVOT maximal flow & 1 & 0.77 & & 1.18 & & \\
velocity (m/s) & 2 & 0.78 & & 1.21 & & \\
& 3 & 0.93 & & 1.04 & & \\
& 4 & 0.65 & & 0.82 & & \\
\hline
\end{tabular}

Explanations: AoV - aortic valve; LVOT - left ventricle outflow tract by plication of the anterior MV ring. This was reflected by statistically unchanged LVOT flow velocities before and after surgery. However, these values were slightly higher than the baseline due to catecholamine support. The AoV remained competent after the operation and did not change its diameter.

In this acute pilot study we were able to demonstrate that partial AML resection completed by corresponding annulus plication is a feasible strategy for restoring MV competency. The proposed procedure resulted in similar outcomes during each experiment. The maneuver did not influence the function and the morphology of the AoV or the flow velocities both through the MV and LVOT. What is noteworthy, the remaining portion of the AML was able to contribute to MV competency. Thus, it seems that the presented technique could be used as a correction method for selected patients requiring removal of a portion of the AML e.g. due to the infective process. The involvement of the AML is usually an indication for MV replacement and constitutes almost one quarter of all prosthetic implantations due to infective endocarditis (2). Moreover, due to the AML size reduction, the implantation of foreign materials could be avoided such as in pericardial patch leaflet reconstruction and/or artificial chordae placement. This may of special interest in case of patients with a growth potential prone to the rapid degeneration and outgrowth of biological or artificial implants. What is the most important, this type of repair concerns only the involved part of the MV and does not jeopardize the rest of the valve which is potentially possible when addressing AML prolapse by the flip-over technique (6).

Surgical techniques concerning AML used to be mainly focused on triangular resection of the A2 scallop (7), retention plasty of A1 and A3 (1) and finally commissure plication (4). In comparison to the presented procedure and to certain operations on PML hitherto carried out, AML interventions are less aggressive in terms of the degree of tissue removal (e.g. AML triangular resection) and MV annulus plication (just around commissures) in order to avoid AoV distortion. The absence of a detrimental influence of partial AML plication on AoV observed in this study could be explained by the dynamic nature of $\mathrm{MV}$ annulus, including its anterior intertrigonal part. Contrary to its remaining parts this segment of the MV annulus expands during the systole to maximize the ejection (5). Thus, it is likely that this ability can be maintained even after the partial plication due to the 
compensatory role of the intact non-operated part of the anterior MV annulus. Secondly, partial plication of the anterior MV annulus may set this segment in permanent "systolic" - i.e. an elongated position by suspension to the commissural part of the annulus. However, this is only a hypothetical explanation and certainly necessitates further studies.

This study is limited by the small number of operated animals which can influence all statistical inferences concerning changes of the studied parameters. The second major drawback of our investigation is its acute character that does not enable insight into long-term outcomes of proposed MV intervention. Certainly, the durability of the presented technique can be a concern. Recently we have reported a pediatric patient presenting congenital pathology complicated by infective endocarditis in which a rescue procedure consisting of partial AML removal and anterior MV annulus plication was performed in order to avoid MV replacement (8). This approach showed excellent 2-year results not complicated by LVOTO sequels. However, a further long-term follow-up is warranted.

Partial AML resection completed by corresponding annulus plication is a feasible and repeatable procedure. It does not negatively influence the functioning of LVOT and AoV. Infective and/or congenital MV involvement in selected patients could be a possible indication for this method of MV reconstruction. Long term experimental assessment concerning MV, AoV and LVOT dynamics and evolution is necessary.

\section{References:}

1. Delmo Walter E. M., Siniawski H., Hetzer R.: Sustained improvement after combined anterior mitral valve leaflet retention plasty and septal myectomy in preventing systolic anterior motion in hypertrophic obstructive cardiomyopathy in children. Eur. J. Cardiothorac. Surg. 2009, 36, 546-552.

2. Feringa H. H., Shaw L. J., Poldermans D., Hoeks S., van der Wall E. E. Dion R. A., Bax J. J.: Mitral valve repair and replacement in endocarditis: a systematic review of literature. Ann. Thorac. Surg. 2007, 83, 564-570.

3.Hetzer R., Delmo Walter E. B., Hübler M., Alexi-Meskishvili V., Weng Y., Nagdyman N., Berger F.: Modified surgical techniques and long-term outcome of mitral valve reconstruction in 111 children. Ann. Thorac. Surg. 2008, 86, 604-613.

4. Kajihara N., Imoto Y., Kan-O M., Sakamoto M., Ochiai Y., Joo K., Sese A.: Clinical results of commissure plication annuloplasty for mitral regurgitation in children. Surg. Today 2009, 39, 110-114.

5. Lansac E., Lim K. H., Shomura Y., Goetz W. A., Lim H. S., Rice N. T., Saber H., Duran C. M.: Dynamic balance of the aortomitral junction. J. Thorac. Cardiovasc. Surg. 2002, 123, 911-918.

6. Mesana T. G., Ibrahim M., Kulik A., Ruel M., Dover K., Nicholson D., Hynes $M .:$ The "hybrid flip-over" technique for anterior leaflet prolapse repair. Ann. Thorac. Surg. 2007, 83, 322-323.

7. Saunders P. C., Grossi E. A., Schwartz C. F., Grau J. B., Ribakove G. H., Culliford A. T., Applebaum R. M., Galloway A. C., Colvin S. B.: Anterior leaflet resection of the mitral valve. Semin. Thorac. Cardiovasc. Surg. 2004, 16, 188-193.

8. WojtalikW., Mrowczynski W., Mrozinski B.: Valve sparing surgery for isolated cleft mitral valve complicated by endocarditis in a child. J. Heart Valve Dis. 2011, 20, 98-99.

Corresponding author: Pawel Antosik, VMD, Dr. of Veterinary Science, Faculty of Veterinary Medicine and Animal Science, Poznan University of Life Sciences, Wolynska 35, 60-637 Poznan, Poland; e-mail: pantosik@ up.poznan.pl 